\title{
Field repair of a severely damaged FG/epoxy fuselage
}

\author{
Piotr Czarnocki ${ }^{1,},{ }^{*}$ Tomasz Zagrajek ${ }^{1}$, and Roman Świtkiewicz ${ }^{1}$ \\ ${ }^{1}$ ITLiMS PW, Nowowiejska 24, 00-665 Warsaw, Poland
}

\begin{abstract}
The paper presents development of a repair procedure for a severely damaged primary laminate structure of a light aircraft, certified in accordance with CS22. The procedure was developed with the assumption that it would be a field repair carried out by people of average manual skills, who have not been trained in repairs of composite structures. To develop the procedure, a typical FG/epoxy laminate glider fuselage was used. The primary objective of the repair was to restore the original fuselage stiffness, while maintaining possibly low stress concentrations resulting from the repairs. To facilitate development of the repair, an FE model was developed. It was parametrized to be useful for design of similar repairs of similar structures. The experimental work done with the use of a real structure allowed the authors to verify numerical simulations, which were found to be correct.
\end{abstract}

\section{Introduction}

The presented repair technology was designed for structural reinstatement of a severely damaged fuselage, including complete break, Fig. 1 (such damage is often caused by a ground loop). Repair of such damage is not allowed by CS23 [1], but CS22 does not impose any limitation concerning this issue and the presented repair technology can be applied to small aircrafts, motor-gliders and gliders certified under CS22. Often, stiffness requirements are the driving factors and therebystrength requirements are also fulfilled. The described repair procedure could be applied to a solid laminate monocoque structure for which critical buckling load was significantly lower than that needed to overrun materials strength. Development of a suitable repair technology is presented later in the paper. The relevant requirements were defined as follows:

- the repair technology should be such that the repair could be done out-of-factory (field repair), but under expert supervision, by people of average manual skills, who have not been trained in repairs of composite structures,

a the repair should restore the original stiffness of the structure not producing excessive stress concentrations in the parent structure,

it should be possible to complete the repair with one-side access,

- the repair could be done either with the help of a vacuum bag or without it,

- the patch should maintain its mechanical properties up to $54^{\circ} \mathrm{C}$.

* Corresponding author: pecz@meil.pw.edu.pl 


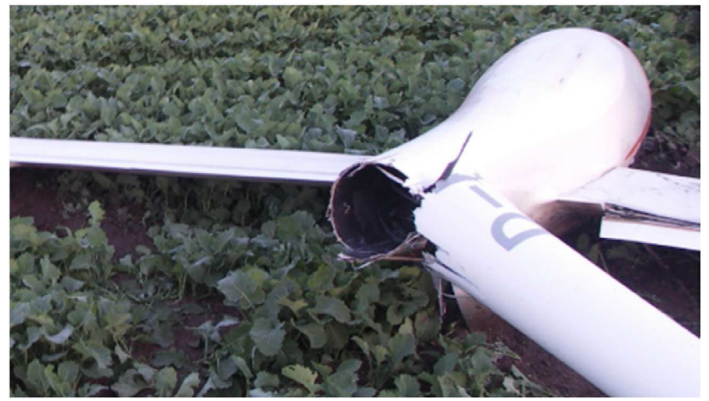

Fig. 1. Typical failure mode of a fuselage caused by a ground loop

Although sufficient repair strength can be proved with tests alone, FE models could be developed to facilitate the repair design process. Such an FE model was concisely presented in this paper. It was parameterized and could thus be applied to solid laminate structures of various geometries. The experimental work presented allowed to validate the model. The Building Block Approach was applied to both experimental work and numerical modelling, Fig. 2. Because of the limitation concerning the lenght of this paper, just the last blocks (the highlighted ones) will be presented. Nevertheless, the preceding tasks were also completed, which involved determination of the necessary material constants, working out and validation of simpler FE models representing repair of a damaged plate (flat patch), as well as tests facilitating the choice of the proper surface preparation method for gluing.

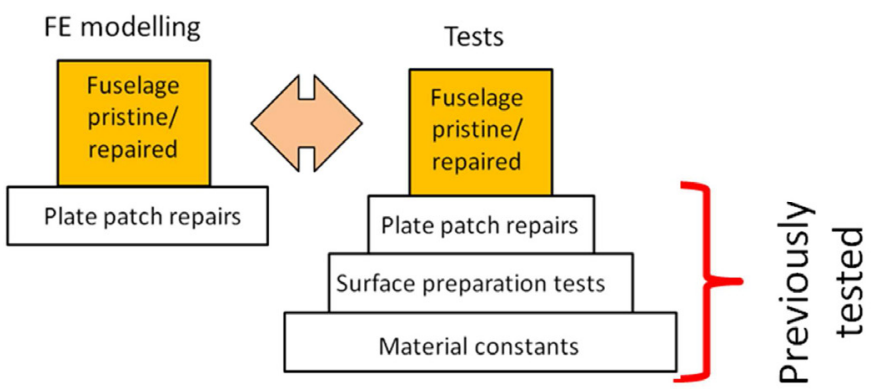

Fig. 2. Applied Building Block Approach

\section{Considered damaged structure}

Still, a large number of composite airframes of motor-gliders, gliders and small airplanes are made of symmetric and UD fabrics impregnated with epoxy resin applying the wet layup manufacturing technique. The presented repair methodology was developed for such structures and for a common lay-up configuration shown in Fig. 3. The important feature of such a lay-up is the arrangement of reinforcement layers. It consists of grouping several reinforcement layers of the same reinforcement directions to form a sublaminate. The structure chosen for the experimental work and the numerical analysis was a 3 meter long section of a fuselage spanning approximately from the trailing edge of the wing to the fin. The fuselage solid laminate monocoque structure, Fig. 3, consisted of two layers of $2 \times 2$ 
twill fabric (Interglas 92125), six layers of UD fabric (Interglas 92145), and one layer of $2 \times 2$ twill fabric (Interglas 92125). The damaged section started $400 \mathrm{~mm}$ behind the front end of the fuselage. Removal of the damaged laminate resulted in a $250 \mathrm{~mm}$ long cut-out encompassing half of the fuselage circumference, Fig. $3 \mathrm{~b}$.
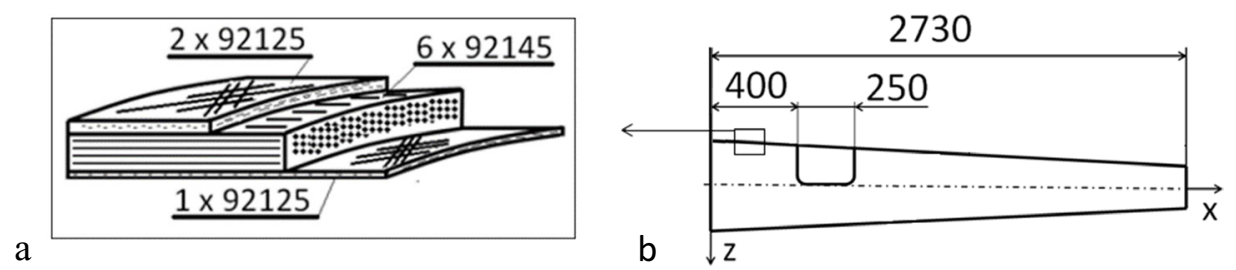

Fig. 3. Considered structure (a) reinforcement lay-up and (b) geometry of the fuselage section and location of the cut out

\section{Repair concept and the patch structure}

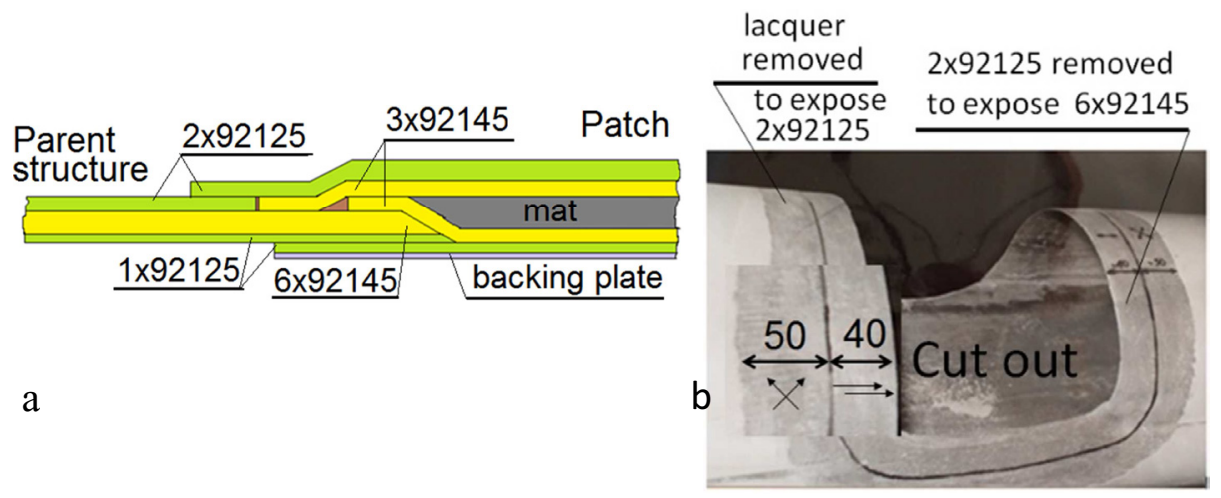

Fig. 4. Patch and parent structure lay-up (a); preparation of the cut-out edges for gluing (b)

A stepped repair was chosen since it is simpler than a scarf one and at the same time offers similar strength [2]. Soft patch technology was chosen for the repair. As recommended [3], restoration of structural continuity in terms of reinforcement directions was attempted wherever possible. To achieve this, the lay-up of the fabric constituting the patch was chosen as shown in Fig. 4a. Sanding was used to prepare the parent structure for gluing. For this purpose, a $50 \mathrm{~mm}$ wide strip of the most outer reinforcement layer (92125 fabric) and $40 \mathrm{~mm}$ wide strip of UD reinforcement (92145 fabrics) of the parent structure were exposed along the cut-out perimeter, Fig. 4b. Also, sanding was applied for surface preparation of the most inner layer of the fuselage shell. The flowchart of the repair procedure ensuring merger of the fabrics with the same reinforcement arrangement is shown in Fig. 5. First, one layer of 92125 fabric was impregnated at the top surface of an $0.5 \mathrm{~mm}$ thick backing plywood plate. Next, with the help of this plate the impregnated but not cured fabric was pressed against the inner surface of the fuselage. For this purpose, a set of bands was used. It was noticed that the band tension affected the camber of the backing plate; thus to minimize possible gaps between the fuselage inner surface and the patch layer, the band tension had to be adjusted. This problem was analyzed with FEM and will be discussed further in the paper. After curing had been completed, the bands were removed and the remaining parts of the patch were formed. It involved placement and 
impregnation of 3 layers of UD 92145 fabric (step\#2), 3 layers of mat forming filling (step\#3), 3 layers of UD 92145 fabric (step\#4) and 2 layers of 92125 fabric (step\#5).
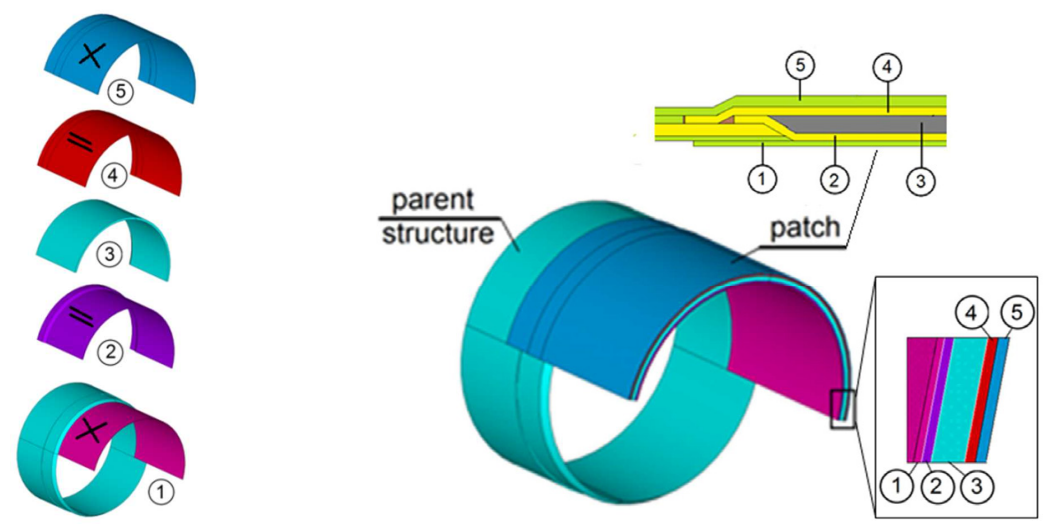

Fig. 5. Flowchart of patch fabrication. The numbers indicate the sequence of actions.

\section{FE modelling}

FE modelling concerned two issues, i.e.:

strength of the bound lines and stiffness of the repaired fuselage, and

$\square$ elimination of possible gaps that could occur between the inner surface of the fuselage and the bottom layer of the patch, since they could weaken the repair.

\subsection{Strength and stiffness analyses}

For this purpose, a commecial FE code ANSYS v.15 [4] was used. The developed model was parametrized to make it versatile and useful for analyzing similar repairs.

\subsubsection{FE model of the fuselage}

A major part of the structure was represented with Shell181 elements, whereas the patch and the $170 \mathrm{~mm}$ wide strip (adjusted to it) of the parent (fuselage) structure were modelled with Solid185 solids. To simulate progressive damage of the bound line (patch-parent structure interface), Conta174 - Targe170 element pairs with the bonded contact option and the cohesive zone bi-linear material model were used. For modelling adhesive joints a cohesive zone material model has been widely applied [5]. The method is not new and its detailed description can be found in [6,7].

The considered bound lines are shown in white in Fig. 6. Load P was applied at the distance of $380 \mathrm{~mm}$ from the $\mathrm{xz}$ plane with the MPC181 element. The opposite end of the fuselage was fixed (all freedom degrees at the nodes in the plane $\mathrm{z}=0$ were removed).

The failure of the bound line was defined in terms of the debonding parameter $\mathrm{d}$.

$$
d=\frac{\Delta-1}{\Delta} \chi
$$

with $\mathrm{d}=0$ for $\Delta \leq 1$, and $0<\mathrm{d} \leq 1$ for $\Delta>1$, where $\Delta$ and $\chi$ are defined as follows: 


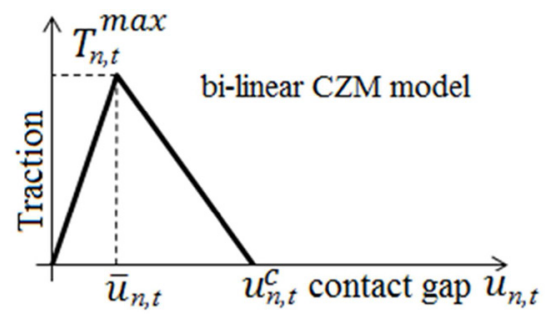

$$
\begin{aligned}
& \Delta=\Delta_{m}=\sqrt{\Delta_{n}^{2}+\Delta_{t}^{2}} \\
& \Delta_{n}=\frac{u_{n}}{\bar{u}_{n}} ; \Delta_{t}=\frac{u_{t}}{\bar{u}_{t}} \\
& \chi=\frac{u_{n}^{c}}{u_{n}^{c}-\bar{u}_{n}}=\frac{u_{t}^{c}}{u_{t}^{c}-\bar{u}_{t}}
\end{aligned}
$$

Subscripts $n$ and $t$ indicate normal and sliding gaps, respectively. For more details, see [1]. $\mathrm{d}=0$ indicated that no debonding had occurred and that the bound line remained intact.
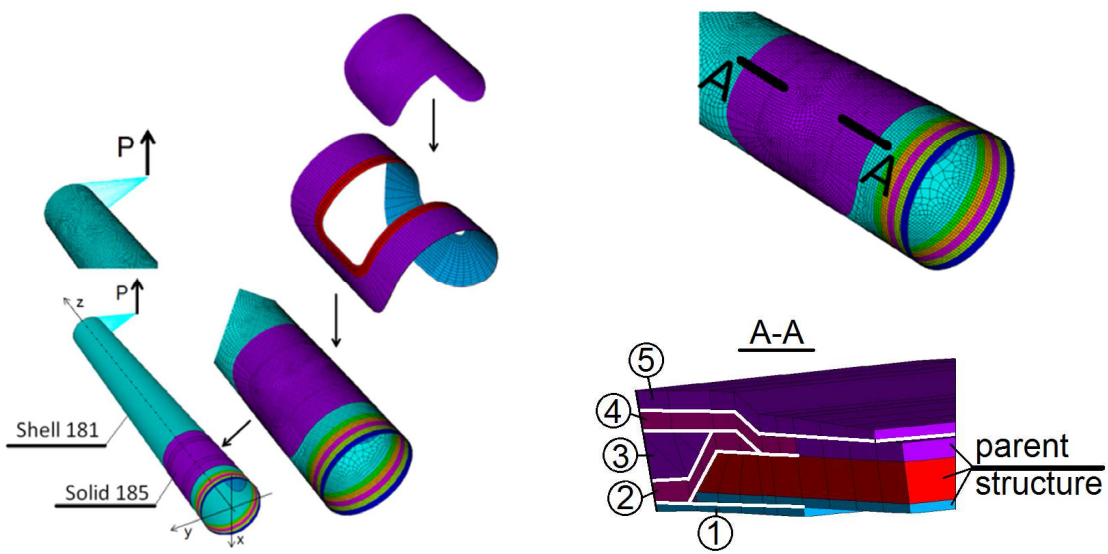

Fig. 6. FE representation of the repaired structure, The white lines (cross-section A-A) indicated the locations of the bound lines simulated with pairs of Conta174-Targe170 contact elements

\subsubsection{Results of the FE analysis}
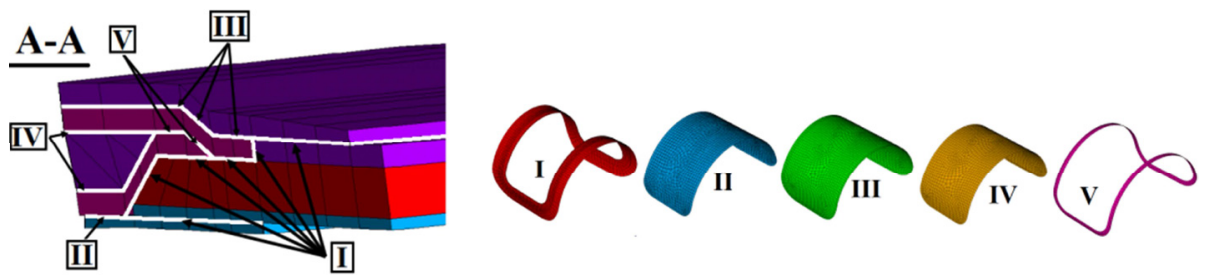

Fig. 7. Areas $\mathrm{I}-\mathrm{V}$ represent the interfaces between the parent structure and the patch, and between the patch sub laminates themselves. The numbers indicate the location of the interfaces

Strength of the bound lines and strength and stiffness of the repaired fuselage. The FE results concerning the debonding parameter $d$ showed that for both the Limit Load (LL) equal to $2.3 \mathrm{kN}$ and the Failure Load (FL) equal to $10.65 \mathrm{kN}$, the debonding parameter $\mathrm{d}=0$ along all the bounded areas, Fig. 7, showed that no damage of the bound lines occurred, so the bound lines remained intact, at least up to failure of the fuselage due to local buckling and the consecutive damage of the fuselage shell at some distance from the patch, Fig. 8. Results of the repair were also analysed in terms of stiffness changes. The deflections $f$ of the ends of the pristine and repaired fuselage section under LL were practically the same. The calculated difference was less than $1 \mathrm{~mm}$ in favour of the latter. In case of twist, the 
results were similar and the difference between the twist of the pristine and repaired fuselage measured at the end of the fuselage was about 0.11 arcminute in favour of the latter, Fig. 9.

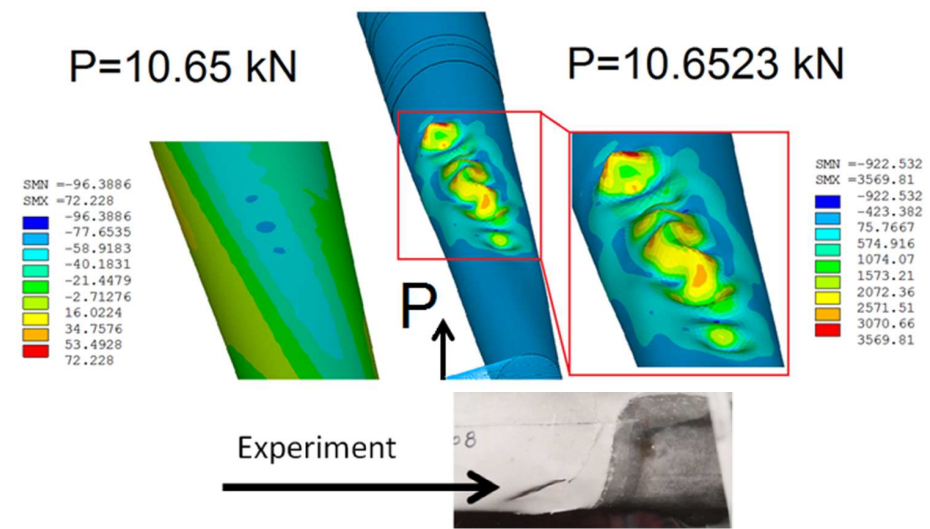

Fig. 8. Pre- and post-buckling stresses corresponding to the loads just before and just after buckling
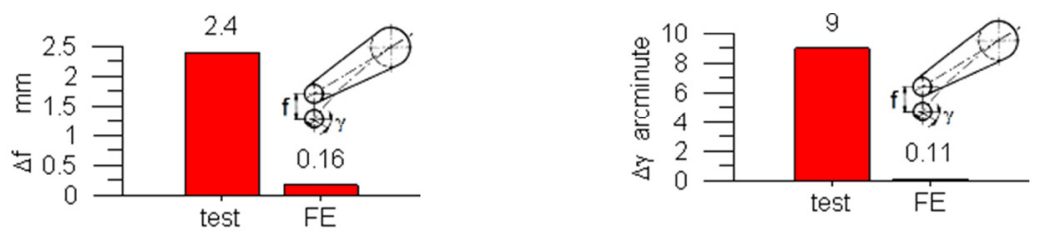

Fig. 9. Differences in the bending f and twist $\gamma$ stiffnesses of the pristine and repaired fuselage, as meassured (test) and calculated (FE)

Gaps between the inner surface of the fuselage and the bottom layer of the patch. The manner in which the plywood backing plate overlapped with the impregnated but not cured reinforcement layer could be pressed against the inner surface of the fuselage with the help of a band set is shown in Fig. 10.
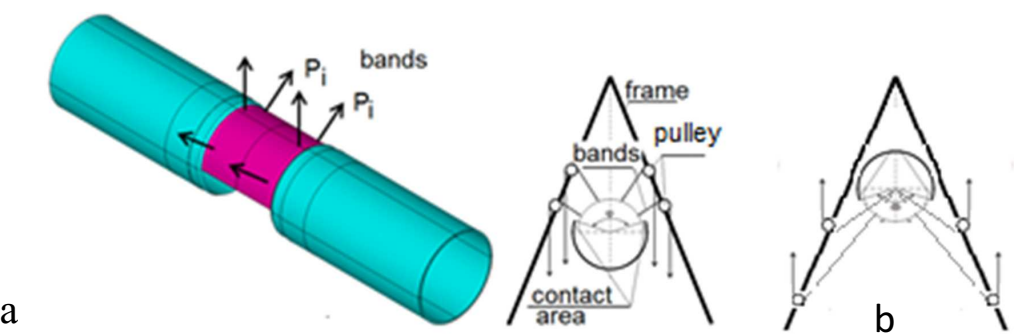

Fig. 10. Application of bands for reducing the gaps between the inner surface of the fuselage and the bottom layer of the patch (a); actual repair: step\#1- fixing the first (lower) backing plate (b)

Such a loading produced double curvature of the backing plate resulting in some gaps between the contacting surfaces. Severity of this phenomenon was analysed with FEM and the results are shown in Fig. 11. It was found that for the chosen band location and the number of bands, the local separation of the contacting surfaces varied with location and the separation went down with decreasing band tension. Also, it was found that to 
reduce the gap opening, the direction of higher stiffness of the backing plate should be paralell to the generator of the fuselage.
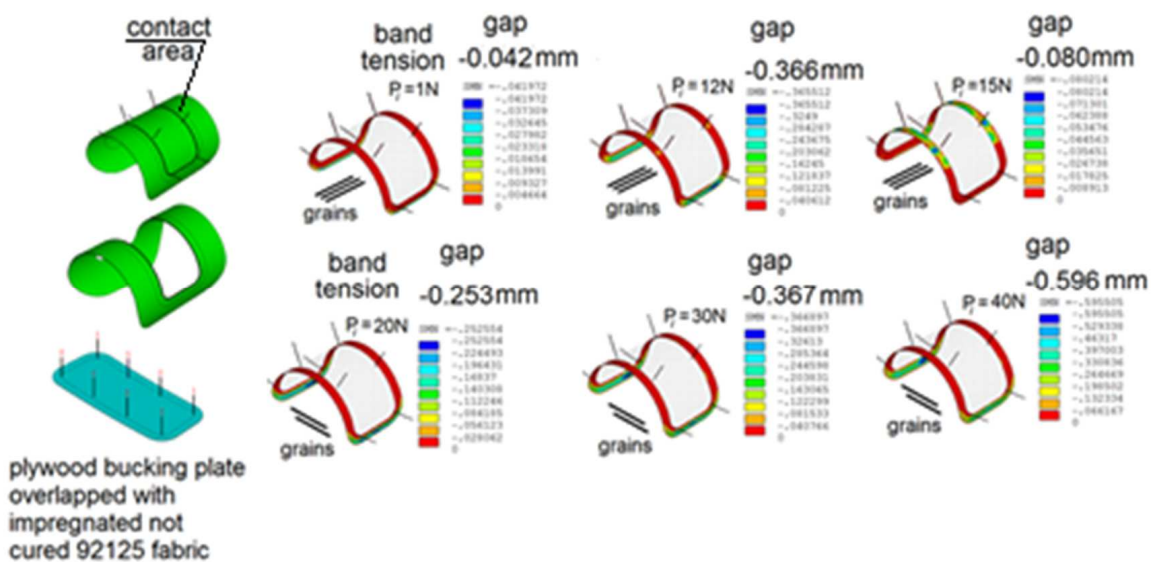
cured 92125 fabric

Fig. 11. Effect of band tension on the gap between the inner surface of the fuselage and the bottom layer of the patch

\section{Experimental work}

A pictorial diagram explaining the manner in which the fuselage deformation was measured and the load was applied is shown in Fig. 12, along with the corresponding experimantal stand.

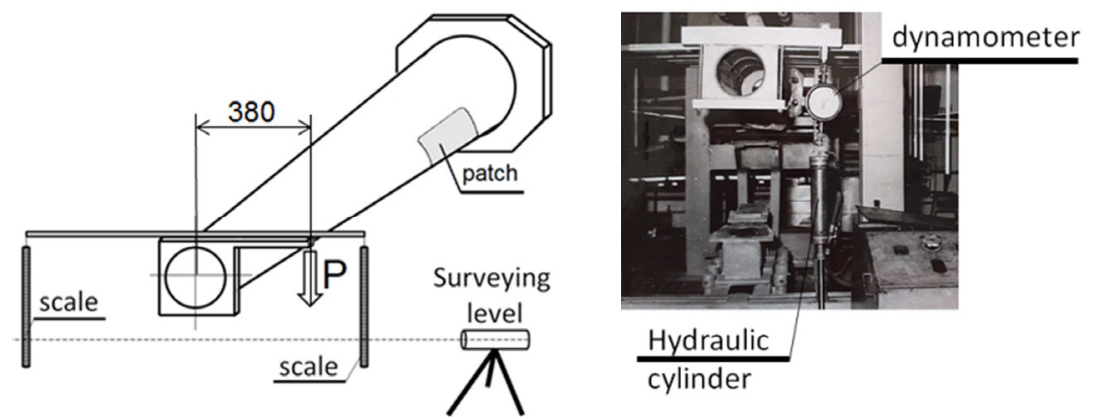

Fig. 12. Experimental stand for investigating the strength and stiffness of the repaired fuselage

The experimentally determined differences (corresponding to LL) between the bending and twist stiffnesses of the pristine and repaired fuselages were equal to $2.4 \mathrm{~mm}$ and 9 arcminutes, respectively, in favour of the repaired one, Fig. 9, and were higher than the calculated deformations. The experimentally determined FL of the repaired fuselage was $10.65 \mathrm{kN}$ and it was negligibly lower than that calculated. The location of the failure and its mode obtained by numerical modelling and determined experimentally were very similar, Fig. 8. Visual inspection of the patch and the one done by tapping did not indicate any damage of the bound lines. 


\section{Practical applications}

The developed technology was applied to a completely broken fuselage, Fig. 13. In general, the patch was fabricated in accordance with the flowchart shown in Fig. 5. The only difference was that after proper alignment of the fuselage parts, the backing plate carrying the impregnated fabric blank was first glued to the lower inner surface of the fuselage and after the curing the upper one was attached as shown in Fig. 5a. Next, steps 2-5 were applied to repair the upper part of the fuselage. After completing the cure, the fuselage was flipped upside down and steps 2-5 were repeated.

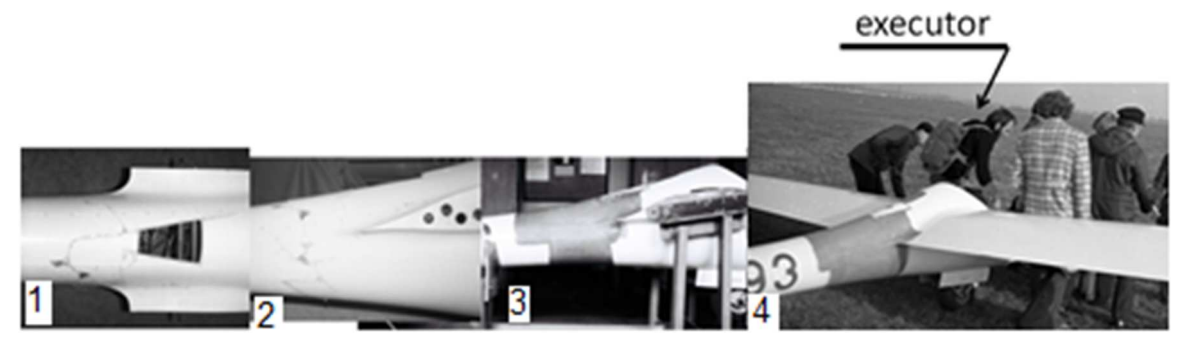

Fig. 13. Actual fuselage damage $(1,2)$ caused by a grand loop, completed repair (3) and the final repair results (4). The patch was intentionally not painted to facilitate monitoring.

\section{Conclusions}

The obtained results are reliable for airframes for which stiffness is a driving factor. In terms of static strength and stiffness, the developed repair procedure met the set requirements. The difference in stiffnesses of the pristine and repaired structure at LL was about $2.4 \mathrm{~mm}$ in case of deflection and 9 arcminutes in case of twist in favour of the repaired structure. The experimentally determined failure load causing local buckling was more than 4 times higher than LL. Some discrepancy occurred between the experimentally determined and calculated differences of the pristine and repaired fuselage deformations at LL. The difference resulting from the calculations was smaller. The difference between the calculated and experimentally determined failure loads was negligible. The failure locus and the failure mode determined by numerical modelling and experiments were very similar. The above suggests that the developed numerical model was reliable. The developed repair technology was verified in the repair of actual damage with respect to its effectiveness and simplicity since the repair was made out of factory by a person without any experience in repairs.

\section{References}

1. EASA CM. : CM-S-005 0/1, 13, (2015)

2. C.H. Vang, V. Venngopal, L. Peng, J.Adheds. 9, 95-112, (2015)

3. M. Niedernhuber, J. Holtmannspotter, I. Ehrlich, Composites Part B 94, 327-337, (2016)

4. Ansys Mechanical APDL Theory Reference, Release v.15.0, 169, (2013)

5. L. F. M. da Silva, R. D. S. G. Campilho, Advances in Numerical Modelling of Adhesive Joints, SpringerBriefs in Computational Mechanics, 17-18, (Springer-Verlag Berlin, Heidelberg, 2012)

6. G. Alfano, M. A. Crisfield, Int. J. Numer. Meth. Engng 50, 1701-1736, ( 2001)

7. E.J. Barbero, Finite element analysis of composite materials using Ansys, 276-284 (CRC Press 2014) 